\title{
Toward fully autonomous structure design based on topology optimization and image processing
}

\author{
Musaddiq Al Ali ${ }^{\mathrm{a}, ~ *}$ \\ ${ }^{a}$ Hiroshima University, 1-3-2 Kagamiyama, Higashi-Hiroshima City, 739-8511, Japan \\ *Corresponding Author: mosadeq007@yahoo.com
}

\begin{abstract}
Design and optimization are recertified advancing exponentially in the recent decades. However, it is important to have human free design CAM CAD process in order to achieve several goals, such as deep space unmanned missions, space mining, and hazardous places maintenance as nuclear reactors, and deep ocean facilities. Other short-term aim is the difficulties of establishing the realistic boundary conditions of complex structure, such as biological organs. This work is designated to implement and test the core of autonomous CAD CAM, i.e. the design process itself. In this paper, image processing is used as the tool for building the design domain for the structural optimization. Structural optimization is performed with topology optimization. Three cases were designed using three objective functions (compliance, single stress, and pnorm stress). The results show the high sensitivity of compliance objective function to cover all the design domain needs. However, the stress of the design parts was not necessarily lower. Pnorm could not give feasible design (the design which covers all the predesigned areas) for one case. Single stress approach gave good stress minimization and achieved feasible design for all the design cases.
\end{abstract}

Keywords: Topology Optimization, Image Processing, Autonomous Design.

\section{Introduction}

Structural design is a mental process based on the designer better judgments and experience. The way of approaching the design problem will vary, according to the designer experience and vision. With the verge of topology optimization by Michelet, and the modern numerical method of structural analysis; Computer-aided design reached new step by fully developing a design based on mathematical optimization principals. Fully computer-based evaluation and design is a goal yet to be reached. Such a goal is a necessity for futuristic frontier transportation missions, such as deep space missions. In such missions, and because of the long distance to the control center, the information from and to the ship is taking a long time and can be blocked due to orbital necessities (In this work quantum communication and informatics are excluded). The space ship may suffer hazardous events which need onsite repair of the damaged part (by an asteroid impact or mechanical fatigue, etc.). In case of manned missions, the damaged part cannot be repaired by crew members because they are theoretically in deep hibernation ${ }^{(1,2)}$. The futuristic deep space missions such as mining ${ }^{(3)}$ can be without a human crew in it for economical purposes. This will necessitate the artificial intelligence (AI) to step in and deals with the designing process and performing the repair by itself. One of the tools that AI can presumably need is an efficient mathematical based designing process. Topology optimization is one of the effective methodologies in mechanical design, especially it is proven to be effective when it associated with additive manufacturing ${ }^{(4,5)}$. Topology optimization ${ }^{(6)}$ generally divided into: of layout optimization and generalized shape optimization. The discretization of the domain into finite parts with a distinctive relation of the parts based on spatial configuration (as finite difference, boxes, element, and volumes). Topology optimization started as a layout problem. The fundamentals of layout optimization is doing the design of specific region (design domain), with fixed traction and support in a point belong to that design space ${ }^{(7)}$. Maxwell in $1869^{(8)}$ studied in detail the traction effect in a frame structure in several papers. Deriving virtual energy formulation to evaluate displacement and applied forces for deterministic and non-deterministic problems, He gave a bound which is the 
difference of compressive and tensile stress within frame members. Michell ${ }^{(9)}$ used Maxwell lemma and did exact analysis formulation and optimization. Feasible optimal design can be achieved due to conditioning based optimization. Hegemier et al ${ }^{(10)}$ review Michell's structure problem for optimal stiffness, creep resistance and natural frequency. Drucker et al (11) applied constant dissipation per unit volume as their study to stress-strain fields and strain energy. Chan ${ }^{(12)}$ study the optimization of static stability of truss structure by developing a technique to determine topographic based strain filed. Dorn et introduced numerical discretization in layout optimization. Bartel $^{(13)}$ in his report, minimized structure weight using sequential unconstrained minimization and Constrained Steepest Descent techniques. Charrett and Rozvany (14) adopted Prager - shield implementation in order to find optimal design criterion considering rigid-perfectly plastic systems under multiple loading. Rozvany and Prager

${ }^{(15)}$ studied optimal design of grillage like continua. Their approach was spatial distribution within confined grillage units. Rossow and Taylor ${ }^{(16)}$ used finite element method as a numerical solution to find the optimum thickness of variable thickness sheets. Potential energy for the elastic sheet in-plane stress assumption was addressed. By introducing holes into plate structure, this work founded shape optimization. Cheng and Olhoff ${ }^{(17)}$ implement finite element method as a numerical solution to optimize the thickness of annual plate with stiffened like approach. Homogenization as averaging method was being adopted in topology optimization a target of the discretized continuous optimality criterion (DCOC) by Bendson et al ${ }^{(18)}$. This work led to adopt the concept of fictitious material by Bendsoe (19) which then derived the famous Solid Isotropic Material with Penalization method (SIMP). In this work, SIMP method is used for optimizing structure with minimum stress concentration. In this paper, image processing is used to construct designing case, and transform it to be designing process for Topology optimization

\section{Autonomous Maintenance Procedure}

For fully integrated maintenance unite, it is valuable to have fully autonomous computer-based CAD-CAM. This will serve a wide variety of engineering necessities. For example, the hazard areas (nuclear reactors, underwater facilities, etc.), and space missions. In order to give the computer, the ability to do free will, for design and rapid prototyping, it needs the ability to recognize the problem, then uses the accumulative designing algorithms to suggest the best design. This step will have followed by robotic rapid prototyping to build and / or mend the defected part. In this work, the target is the image processing step, mathematical modeling, and the examining topology optimization as a designing method.

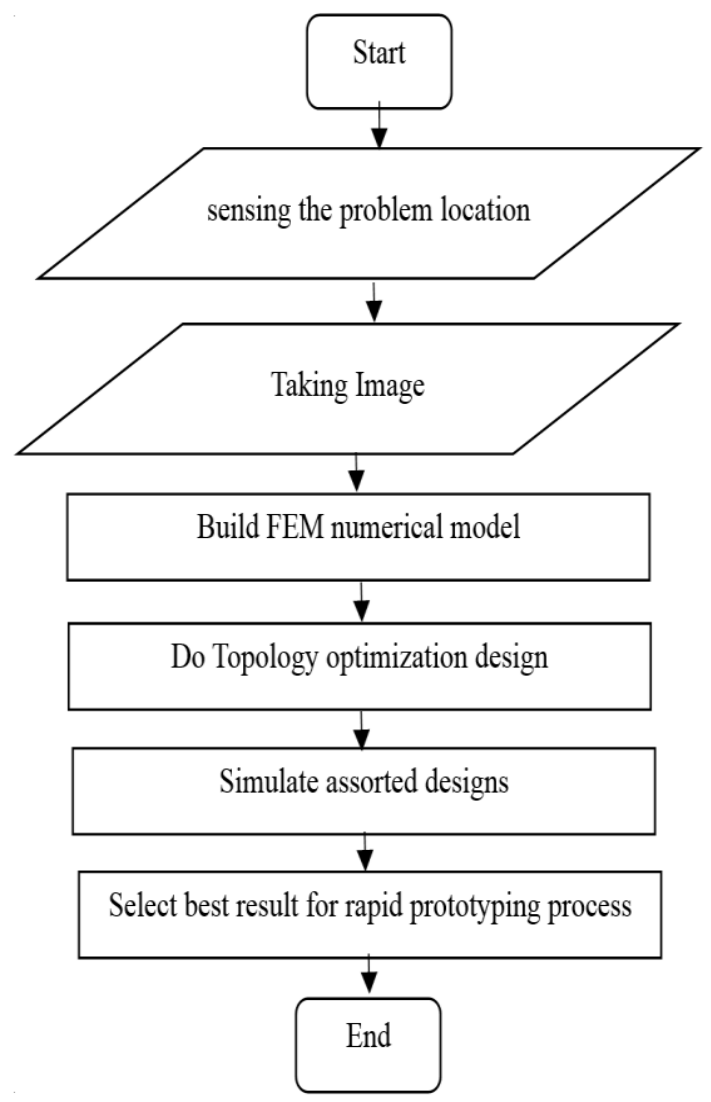

Fig. 1. Autonomous design Algorithm.

\section{Image Processing}

First $\mathrm{s}$ step is to take a picture of the space that the structure should be built in; so, the design domain will be created using image processing. Image processing also will be implemented to describe the places of traction and support. The filters of image processing may vary according to the complexity of signal analysis and the type of the charge couple device that take it. Digital images are consisting of pixels. Pixel is the building block of the spatial distribution of the image. Each pixel has Intensity (Grayscale)/ Intensities (colored). Color is represented in term of the fundamental computer information building 
block i.e. binary digits (bits). In the standard byte ( 8 bits) $)^{(20)}$, it has 28 values of the color scale. Color image in a simple form consists of various intensity values of the three Maxwell fundamental colors (Red, Green, and Blue). In the problem which is represented in this work; color image is not been addressed. The only information that is needed to be obtained is: if there are materials or not. In case of no materials, the computer will set it as the design domain. The material existing parts will be assumed to have the boundary conditions of the problem.

\section{Topology Optimization}

Topology Originally, structural optimization is a matter of a determinably functional analysis based on a noticeable physical model. With increasing of adopting mathematical modeling in a real-world application associated with the advancement of mathematics (understanding and technique), the various methods are introduced to include the previously Underdetermined problems. Virtual work concept expanded the scope of solving the real-world cases $^{(21)}$. Structure optimization tends to be known as parametric and non-parametric optimization. Parameters usually taking the aspects of topographical representation domain in terms of well approximated mathematical model related to the constraints and the optimality criteria. Heuristic and metaheuristic methodologies are used effectively for the parametric optimization. Sizing optimization is an example of parametric optimization, such that cross-section, size of desired material, and/or holes could directly have linked to stress minimization problem. Finding the optimal material representation in space for best structural design is not an easily represented in the scope of the known quantities that represent the problem such as masses, loads, reactions, etc. The meaning of the easiness here is to link the topology design directly to the known values (constraints) and make universal parametric objective for it. This lead to the other optimization methodology which is non-parametric optimization. Examples of such method are topology optimization (layout and shape optimization). Topology optimization promoted as the methodology of new possibilities, with the purity of mathematical logic. The aspects of the design are based on the mathematical optimization of a known state space or higher order set of objective criteria. Such design should obey only the necessity of satisfying the optimizing process which gives theoretically the best feasible (solution bounded by a period), optimal solution possible). optimization as non-parametric design methodology uses cascade approximations stating from the design variables Such as SIMP method and through the discretization method, objective criteria, and ended with the design variables update. Topology optimization using SIMP method is been adopted by many companies in their commercial software (Such as Autodesk, ANSYS, COMSOL, Dassault, Altair, LSTC, Quint, and CAESS) so it has proven its adaptability and relatability for the engineering problems. Topology optimization according to the objective criteria are compliance based (Equation (1)), and stress based (Equation (2)). In this work, MATLAB programs were written to perform topology optimization

$$
\begin{aligned}
& \lim _{\text {volf } \rightarrow 0} x\left\{\Gamma\left(\Omega \cap \Omega_{\text {mat }}=0\right)\right\} \\
& \min ., \mathbf{F}^{T} \mathbf{u} \quad \mid E_{\min \rightarrow 0}+x^{q}\left(E_{\min \rightarrow 0}+E_{\text {mat } \rightarrow 1}\right) \\
& \text { s.t. } \int_{\Omega_{d}} \rho d x \leq V_{d}, 0<x_{\min }<x_{e}<1 \quad \forall x \in \Omega_{d} \\
& \min f=\left\{\begin{array}{c}
\rho^{p-q}\left(\frac{\sigma_{\text {avg }}}{\sigma_{\text {yield }}}\right): q p-\text { approach } \\
\sqrt[p]{\sum_{i=1}^{n}\left|\frac{\sigma_{\text {avg }}}{\sigma_{\text {yield }}}\right|^{P}}: p-\text { norm approach }
\end{array}\right. \\
& \text { s.t. } \int_{\Omega_{d}} \rho d x \leq V_{d}, 0<x_{\min }<x_{e}<1 \quad \forall x \in \Omega_{d}
\end{aligned}
$$

Where, $\mathbf{F}^{T} \mathbf{u}$ is the compliance objective function.

Power (q)that satisfy the condition of two dimensions (22) (2.8-3.7).

The same points of promotion for topology optimization are actually pointing a serious aspect to be careful at. For example, deriving sensitivity for gradient-based optimization is not an easy task especially with complicated and cascade functions. Numerical sensitivity may $\mathrm{ab}$ an option. However, numerical sensitivity consumes a lot of time and computational power. The good results also need high resolution to get so high consumed power is inevitable. Not to mention the discretization as well as the simplification (which is needed and highly accepted) will not give the full picture of the physical phenomena, and the accurate solution for it. On the other hand, the mathematical chaos which is highly anticipated in the complex model can leads to failure in the whole computation operation. All of this can be categorized as the mathematical challenge of the topology optimization. Objective function design is a challenge has been mentioned in mathematical optimization section. A well-designed objective function may give strange optimal results which are by the original mathematical model of the 
problem is a highly suspicious solution. For example, the topology optimization of heat conduction which produces the famous tree-like model ${ }^{(23)}$. According to the propagation of heat in solid, the wavefront should be moving in an even distribution. The tree model seems even in distribution, yet mas is not distributed evenly in all directions to satisfy the anticipated best distribution. Yan et al ${ }^{(24)}$ presented another model showing the validity of these suspicions. Another example is the topology optimization of discretized Maxwell equation. Despite the fact that, the discretization itself is limited for the advanced problem which such non-parametric i.e. layout and shape optimization (Topology optimization) are needed; the suggested criteria are also affected seriously on producing at least $80 \%$ accurate design which satisfies the physics of electromagnetic phenomena. For example, the optimization of magnetic circuitry ${ }^{(25)}$. The design has been produced are mathematically challenging, yet the real application is predicted to finds them unsatisfying. This is the Human factor of the topology optimization challenges. Another aspect of topology optimization challenge is the discretization itself. In the produced design, areas on non-feasible design may occur ${ }^{(26)}$ as shown in Figure (2). This is varying with using high order degree of freedom (Figure (3). To solve this problem, extra filtering has usually been used. The filtering in relying on the designer experience and better Judgment.

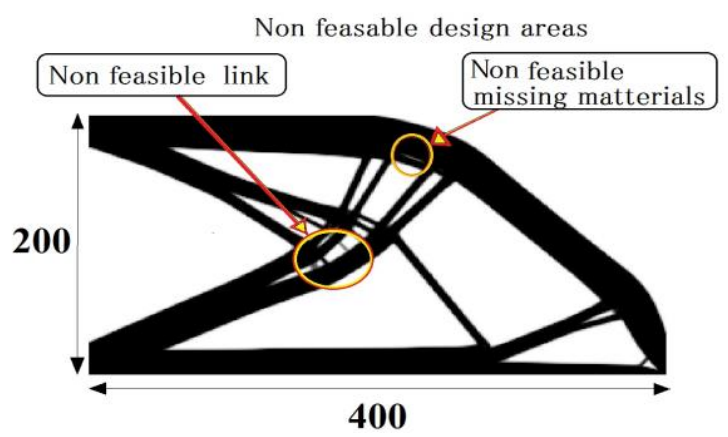

Fig. 2. Objective function noise

Topology optimization is a methodology uses the full potential of the advancement in computation power and methodologies. This will lead to the possibility of fully autonomous design. For example, the non-man mission can do design or repair itself based on such methodology, aided with advanced computer vision and rapid prototyping. Topology optimization may be the only way in certain application such as the inner design of custom orthopedic cause it is based on the geometrically complex system. It is hard enough to perform accurate analysis considering the real physical model of the organ.

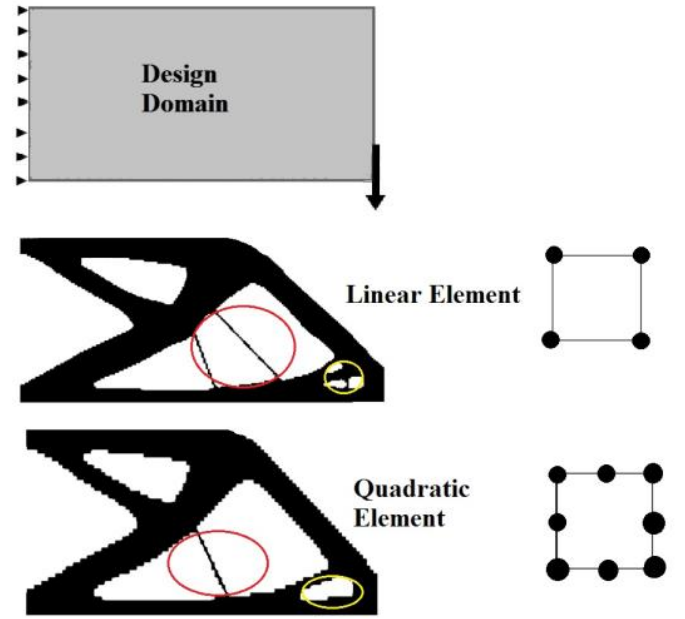

Fig. 3. Design feasibility for the used element

This will leave only topology optimization as the main method for revolutionary and satisfying design because it is has been mentioned before; it is relying on the pure logic of mathematics.

\section{Conventional method versus image processing imp limitation}

Modern physical problems have become complex, making continuous mathematical models are not quite practical to be used. The numerical solution is used to simplify the modeling. Boundary conditions and constraints are represented in the fixed field act on the elemental nodes. The element local coordinate will be transferring to be a part of the generalized stiffness matrix. The numbering has certain complexity according to the grid design.

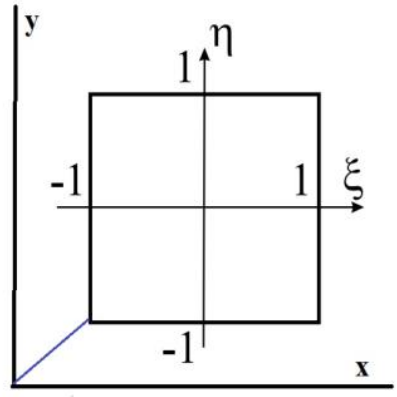

Fig. 4. Local to global element transfer concept

The following case of numbering is being implemented in this work. The boundary conditions needs to enter as a function or to be entered manually, node by node. With increasing the resolution of the and the complexity of the problem, the boundary condition assigning will consume amount of efforts which can be invested in with other 
aspect of the designing process. Non-design domain in the other hands needs to enter the elemental density also as a function or element by element. The complex shape cannot be presented in an equation, so it needs in conventional method, an element by element building process. For example, the human femoral head optimization problem (Figure.6) is a complex problem to set using the conventional human based boundary setting and design/ non-design element identifications.

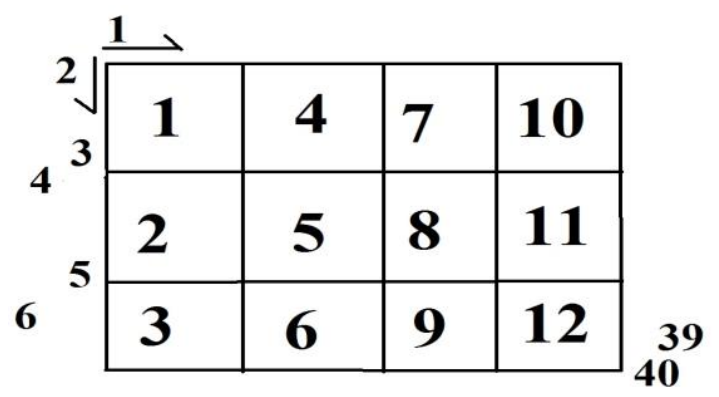

Fig. 5. Finite element mapping example

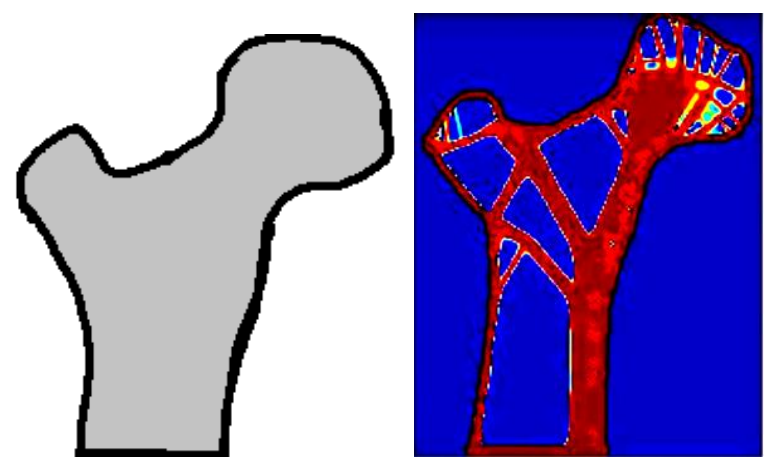

Fig. 6. Complex problem example, Human Femoral head topology optimization example

\section{Numerical Example}

As a pilot study, a simple code written in MATLAB to import a picture of several design problems. Topology optimization was performed to do the design. The images were imported to MATLAB, and the design domain is being set according to the size of the picture. The white areas are the areas to have the design. Optimality criteria was chosen as optimization methodology for compliance, single stress, and pnorm functions. Sensitivity analysis was performed, using the adjoint method. Design domain and boundary conditions were implemented by image processing. Such that the right area having the load while the left boundaries are the fixation. Figure (7) shows the numerical examples that been chosen and the converting process to be finite element design domain.

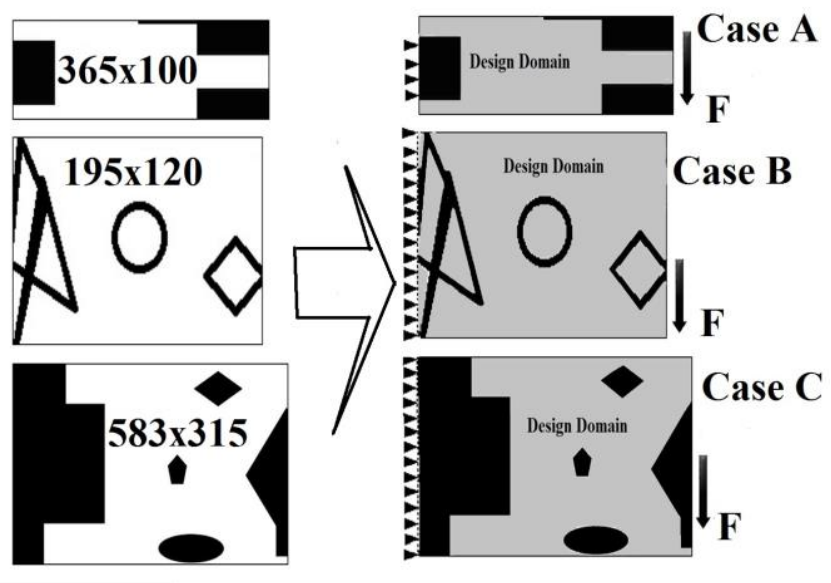

Fig. 7. Design domain analysis using Image processing.

\section{Results and Discussions}

The results of optimization are being divided into 3 groups, the first group is the compliance-based design, the second group is the single stress, and the third group is for pnorm function. The produced topology optimization is shown in figures 8,9 and 10 .
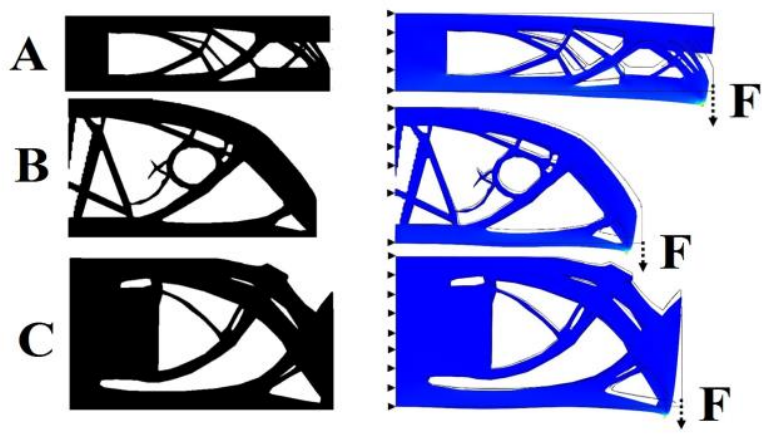

Fig. 8. The resulted Compliance function design
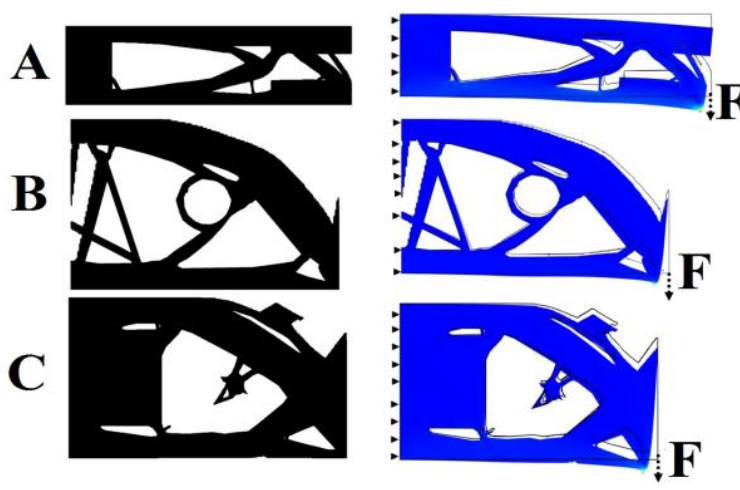

Fig. 9. The resulted single stress function design 


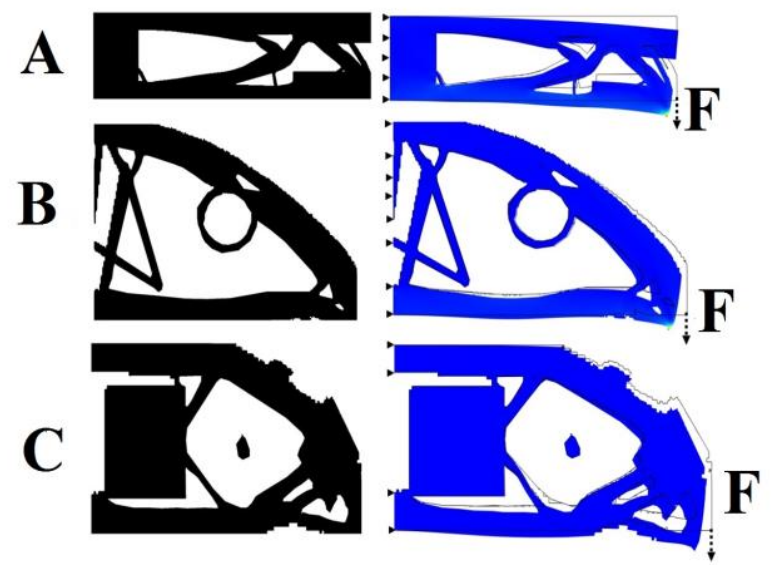

Fig. 10. The resulted pnorm stress function design

All Stress based topology optimization was not sensitive enough to the design domain. So, in the case of pnorm stress, the central area of case $\mathrm{C}$ was not connected. This is happening because the pnorm stress attempt to aggregate the stresses, so overall stress criteria are being optimized. From stress since, the central area is a non-feasible design area, such that addressing it will maximize the overall stress. In the other hands, single stress gave good feasibility to all design examples. It shows good linking for all dark areas (the pre-design domain). Table 1. Shows the maximum von Mises stress for the design cases.

Table 1. von Mises stress results of optimization examples.

\begin{tabular}{|l|l|l|l|}
\hline Structure & A & B & C \\
\hline Compliance & 3.7 & 4.8 & 7.2 \\
\hline Local Stress & 3.7 & 1.23 & 5.3 \\
\hline pnorm & 3.7 & 4.7 & 2.4 \\
\hline
\end{tabular}

The stress minimization for pnorm and single stress is varying with the design case. Pnorm is considered to stable methodology, but sometimes, as in case B in Table 1. Cannot give the best minimization of stress.

\section{Conclusion}

Image processing can be considered as a good input method for the design domain comparing to manual conventional method. In biological cases, it will be impossible to use the conventional method due to domain complexity. Topology optimization shows good adaptation to the input images. Compliance function shows good covering for the design and the non-design domain. Pnorm gave good stress minimization yet it did not cover all the design criteria in case C. the methodology of structural optimization with topology optimization aided image processing showed feasible design for the most design cases. In order to achieve assure feasible design, Multiobjective criteria are suggested to be used.

\section{Acknowledgment}

The author would like to thank Mr. Amjad Y. Sahib, for his valuable help and advice in the matter of image processing.

\section{References}

(1) T. Cockett and C. C. Beehler, "Protective effects of hypothermia in exploration of space," JAMA, vol. 182, pp. 977-979, 1962.

(2) R. D. Launius, "Can we colonize the solar system? Human biology and survival in the extreme space environment," Endeavour, vol. 34, pp. 122-129, 2010.

(3) M. C. He, H. Xie, S. Peng, and Y. Jiang, "Study on rock mechanics in deep mining engineering," Chinese Journal of Rock Mechanics and Engineering, vol. 24, pp. 2803-2813, 2005.

D. Brackett, I. Ashcroft, and R. Hague, "Topology optimization for additive manufacturing," in Proceedings of the solid freeform fabrication symposium, Austin, TX, 2011, pp. 348-362.

R. Ponche, O. Kerbrat, P. Mognol, and J.-Y. Hascoet, "A novel methodology of design for Additive Manufacturing applied to Additive Laser Manufacturing process," Robotics and Computer-Integrated Manufacturing, vol. 30, pp. 389-398, 2014.

(6) G. Rozvany, "Topology optimization in structural mechanics," Structural and Multidisciplinary Optimization, vol. 21, pp. 89-89, 2001.

H. L. Cox, The Design of Structures of Least Weight: International Series of Monographs in Aeronautics and Astronautics: Solid and Structural Mechanics vol. 8: Elsevier, 2014.

(8) J. C. Maxwell, The Scientific Papers of James Clerk Maxwell vol. 2: University Press, 1890.

(9) A. G. M. Michell, "LVIII. The limits of economy of material in frame-structures," The London, Edinburgh, and Dublin Philosophical Magazine and Journal of Science, vol. 8, pp. 589-597, 1904. 
(10) G. Hegemier and W. Prager, "On michell trusses," International Journal of Mechanical Sciences, vol. 11, pp. 209-215, 1969.

(11) D. C. Drucker and R. Shield, "Design for Minimum Weight," BROWN UNIV PROVIDENCE RI1956.

(12) A. Chan, "The design of Michell optimum structures," College of Aeronautics Cranfield1960.

(13) D. L. Bartel, "Optimum design of spatial structures," IOWA UNIV IOWA CITY DEPT OF MECHANICS AND HYDRAULICS1969.

(14) D. Charrett and G. Rozvany, "Extensions of the Prager-Shield theory of optimal plastic design," International Journal of Non-Linear Mechanics, vol. 7, pp. 51-64, 1972.

(15) G. Rozvany and W. Prager, "Optimal design of partially discretized grillages," Journal of the Mechanics and Physics of Solids, vol. 24, pp. 125-136, 1976.

(16) M. Rossow and J. Taylor, "A finite element method for the optimal design of variable thickness sheets," Aiaa Journal, vol. 11, pp. 1566-1569, 1973.

(17) K.-T. Cheng and N. Olhoff, "An investigation concerning optimal design of solid elastic plates," International Journal of Solids and Structures, vol. 17, pp. 305-323, 1981.

(18) M. P. Bendsøe and N. Kikuchi, "Generating optimal topologies in structural design using a homogenization method," Computer methods in applied mechanics and engineering, vol. 71, pp. 197-224, 1988.

(19) M. P. Bendsøe, "Optimal shape design as a material distribution problem," Structural and multidisciplinary optimization, vol. 1, pp. 193-202, 1989.

(20) J. C. Russ, The image processing handbook: CRC press, 2016.

(21) A. P. Boresi, R. J. Schmidt, and O. M. Sidebottom, Advanced mechanics of materials vol. 6: Wiley New York, 1993.

(22) M. P. Bendsøe, O. Sigmund, M. P. Bendsøe, and O. Sigmund, Topology optimization by distribution of isotropic material: Springer, 2004.

(23) M. P. Bendsoe and O. Sigmund, Topology optimization: theory, methods, and applications: Springer Science \& Business Media, 2013.

(24) S. Yan, F. Wang, and O. Sigmund, "On the non-optimality of tree structures for heat conduction," International Journal of Heat and Mass Transfer, vol. 122, pp. 660-680, 2018.

J. Lee, E. M. Dede, D. Banerjee, and H. Iizuka, "Magnetic force enhancement in a linear actuator by air-gap magnetic field distribution optimization and design," Finite elements in analysis and design, vol. 58, pp. 44-52, 2012.

(26) N. Olhoff, M. P. Bendsøe, and J. Rasmussen, "On CAD-integrated structural topology and design optimization," Computer Methods in Applied Mechanics and Engineering, vol. 89, pp. 259-279, 1991. 